\title{
Integration and simulation of microfluidic platforms and terahertz time-domain spectroscopy systems
}

\author{
Rasha Al-Hujazy \\ School of Engineering \\ University of Guelph \\ Guelph, Canada \\ ralhujaz@uoguelph.ca
}

\author{
Christopher M. Collier \\ School of Engineering \\ University of Guelph \\ Guelph, Canada \\ ccollier@uoguelph.ca
}

\begin{abstract}
This work serves as a design tool for the development of integrated terahertz time-domain spectroscopy systems with microfluidic platforms. The emission of terahertz radiation from a GaAs $\mathrm{THz}$ antenna is simulated. This simulation involves a semi-classical computational model with white noise effects used to control the dynamic range of the system. The maximum measurable frequency for the overall integrated system is strongly influenced by the sample thickness of the microfluidic platform and the dynamic range of the terahertz time-domain spectroscopy system.
\end{abstract}

\section{Keywords-biosensing; microfluidics; terahertz}

\section{INTRODUCTION}

Ultrafast science and measurement of terahertz (THz) electromagnetic radiation is a rapidly-growing research area with many applications [1-7]. Terahertz electromagnetic radiation occupies a unique section of the electromagnetic spectrum, being $0.1-10 \mathrm{THz}$. This $\mathrm{THz}$ electromagnetic radiation has important applications in the fields of security [8], communications [9], quality control [10], and biomedical spectroscopy [11].

One of the most important tools that has been enabled through $\mathrm{THz}$ electromagnetic radiation is $\mathrm{THz}$ time-domain spectroscopy (THz-TDS). Terahertz time-domain spectroscopy [12] has been applied to various biomedical applications, including genome analysis [13], oncology [14], and proteomics [11]. In THz-TDS, absorptive and refractive material properties are detected in pulses of $\mathrm{THz}$ radiation with extremely short duration $(<1 \mathrm{ps})$ through changes to amplitude and phase, respectively. Measurements are performed with and without a sample and compared. For analyses of vibrational and rotational modes, THz-TDS performance is at or beyond that of traditional methods such as Fourier transform spectroscopy [15]. Additionally, $\mathrm{THz}$ electromagnetic radiation is non-ionizing, making THz-TDS an appealing option compared to X-ray and other spectra [16].

Despite the above advantages, THz-TDS systems have critical design parameters which should be taken into account for use in modern biomedical devices. This is especially true for integration with microfluidic devices [17-20]. These limitations come from the strong absorption of liquids at $\mathrm{THz}$ frequencies. With this strong absorption in liquids, THz-TDS microfluidic systems must be made with sufficiently small microfluidic platform thickness and sufficiently high dynamic range, to ensure that spectroscopy is properly performed over the full bandwidth of the THz-TDS system [21].

This paper addressed the knowledge gap between microfluidic platforms and THz-TDS systems. We present a comprehensive investigation of microfluidic platform thickness and dynamic range for a THz-TDS microfluidic system. Data analysis is implemented through a semi-classical computational method [22] with white noise incorporated into the simulation. An increasing noise amplitude is used to examine the effects of THz-TDS dynamic range. The maximum measurable frequency, $f_{\max }$, of the system is found for numerous combinations of input parameters, being dynamic range and microfluidic platform thickness.

\section{THEORY}

A THz-TDS microfluidic system is shown in Fig. 1. Here, an ultrafast laser pulse pumps a THz emitter to generate a reference $\mathrm{THz}$ pulse. Upon passing through the microfluidic platform, the reference THz pulse, $E_{\mathrm{R}}(f)$ becomes the THz sample pulse, $E_{\mathrm{S}}(f)$.

The electric field of the $\mathrm{THz}$ pulse in the far-field can be expressed [23] as

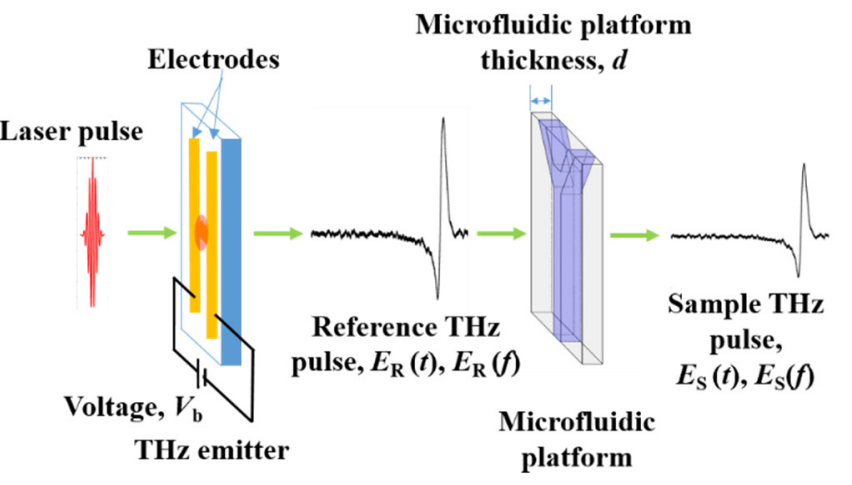

Figure 1. Microfluidic-based THz-TDS system 


$$
E(z, t)=\frac{L_{y}}{8 \pi \varepsilon_{o} c^{2} z} \frac{d}{d t} \int_{0}^{L_{x}} k_{n}(x, t) d x
$$

where $L_{y}$ is the gap width in the $y$-direction, $L_{x}$ is the gap width in the $x$-direction, $\varepsilon_{0}$ is the permittivity of free space, $c$ is the speed of light, and $k_{n}(x, t)$ is the surface current of electrons. $=$ (The electric field is simulated according to the semi-classical computational method of Rodriguez and Taylor [22].)

The semiconductor electromagnetic equations for the $\mathrm{THz}$ emitter accomplishes a noise free radiation, the white noise (which is dominant over other noise sources at high $\mathrm{THz}$ frequencies) is added and defines the noise floor of the $\mathrm{THz}$ emitter measurements [24]. The equation of the reference $\mathrm{THz}$ pulse is

$$
E_{R}(t)=E(z, t)+A_{n} n(t)
$$

where $A_{n}$ is the noise amplitude, and $n(t)$ is the uniform random noise signal (centered about zero, peak-to-peak spread made the same as $\mathrm{THz}$ pulse amplitude). A Fourier transform of the generated pulse can be used to find the dynamic range for different noise conditions. The maximum absorption coefficient for a THz-TDS system is

$$
\alpha=\frac{1}{d} \ln \left[D R \frac{4 n}{(n+1)^{2}}\right]
$$

for refractive index of $n$ [25].

\section{RESULTS}

The simulation is run with standard operation conditions of $50 \mathrm{~mW}$ average power in the laser pulse and external bias of 0.17 $\mathrm{V} / \mu \mathrm{m}$. The effect of noise amplitude, $A_{n}$, on the reference $\mathrm{THz}-$ TDS pulse is investigated by increasing the noise amplitude from zero to $1 \mathrm{E}-3,1 \mathrm{E}-2,1 \mathrm{E}-1,1 \mathrm{E} 0$, and $1 \mathrm{E} 1$. The resulting reference THz pulses in the time domain are shown in Fig. 2 for progressively increasing $A_{\mathrm{n}}$ values. The same $\mathrm{THz}$ pulses in the frequency domain are shown in Fig. 3 for progressively decreasing dynamic range values of $\mathrm{DR}=5.8 \mathrm{E} 3,5.7 \mathrm{E} 3,2.4 \mathrm{E} 3$, $3.0 \mathrm{E} 2,2.8 \mathrm{E} 1$, and $2.8 \mathrm{E} 0$. It is clear that as noise amplitude increases, dynamic range decreases.

By finding the intersection of the maximum absorption coefficient with a known water absorption coefficient curve, $\alpha_{\mathrm{W}}$, the maximum measurable frequency can be found. This is shown in Fig. 4(a) for $d=120 \mu \mathrm{m}$ and DR $=2.4 \mathrm{E} 3$. For decreased DR or increased $d$, the $\alpha$ curve will shift down, forcing maximum measurable frequency to a lower value. For increased DR or decreased $d$, the $\alpha$ curve will shift up, forcing maximum measurable frequency to a higher value. Given this behaviour, the various dynamic ranges shown in Fig. 3 can be used in conjunction with the absorption coefficient of water, being $\alpha_{\mathrm{W}}=$ $61 f+54 \mathrm{~cm}^{-1}$, to find the corresponding maximum measurable frequency.

Ultimately, the maximum measurable frequencies can be collected for a variety of simulated conditions for microfluidic platform thickness and dynamic range. This is shown in Fig. 4(b) which displays a three-dimensional surface plot that shows the maximum measurable frequency versus dynamic range and microfluidic platform thickness. As described previously, one

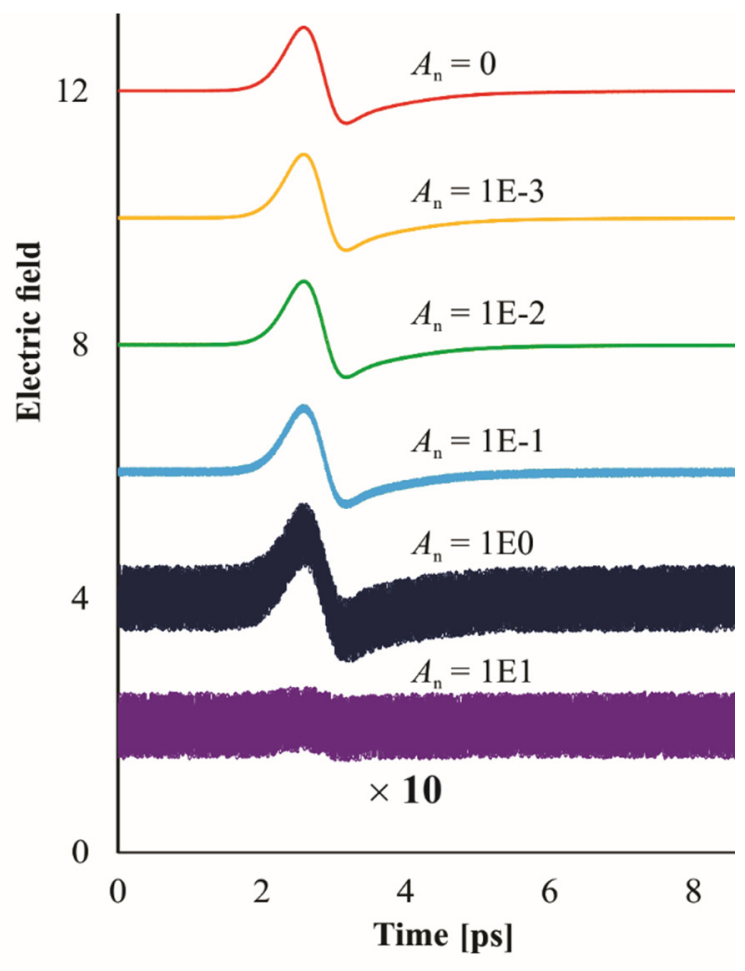

Figure 2. Simulated $\mathrm{THz}$ pulses in the time domain with different noise amplitudes.

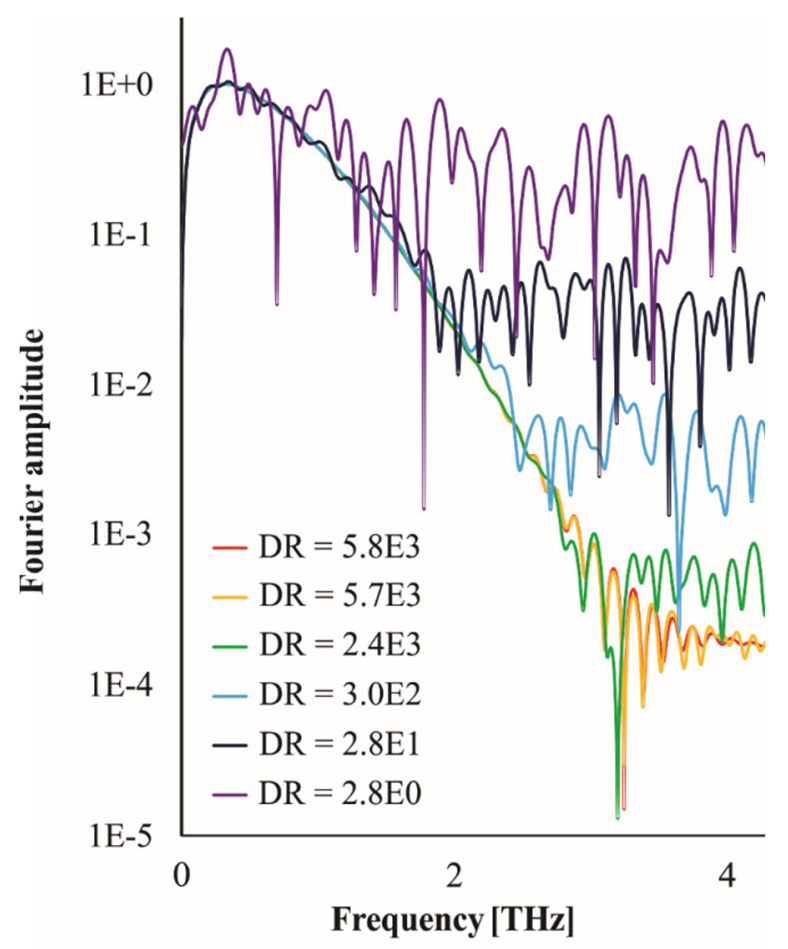

Figure 3. Simulated $\mathrm{THz}$ pulses in the frequency domain with different dynamic ranges (corresponding to the noise amplitudes from Fig. 2). 


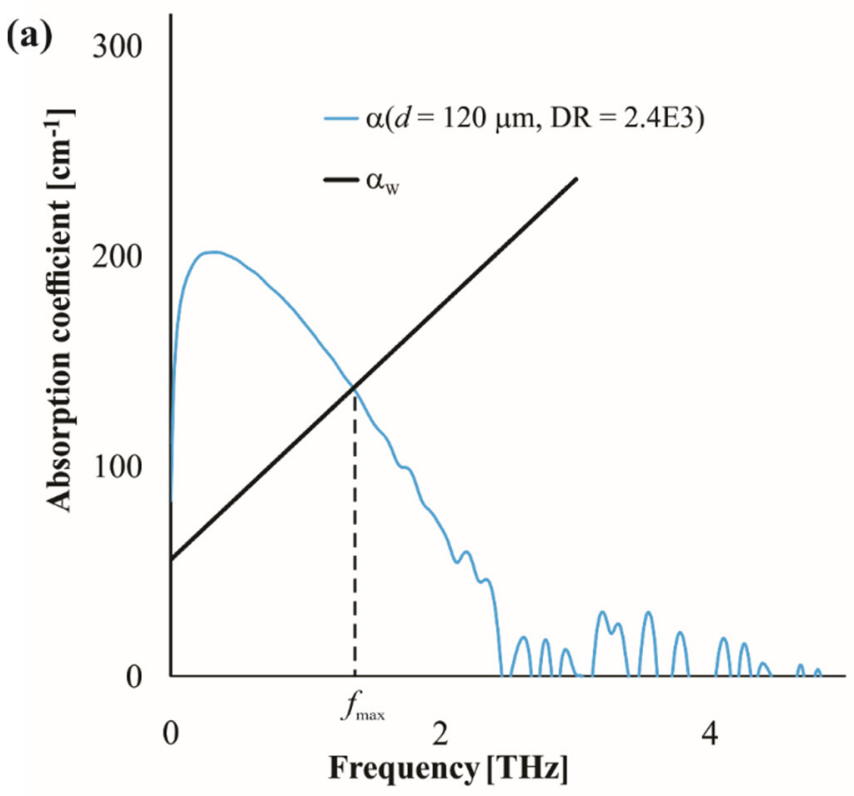

(b)

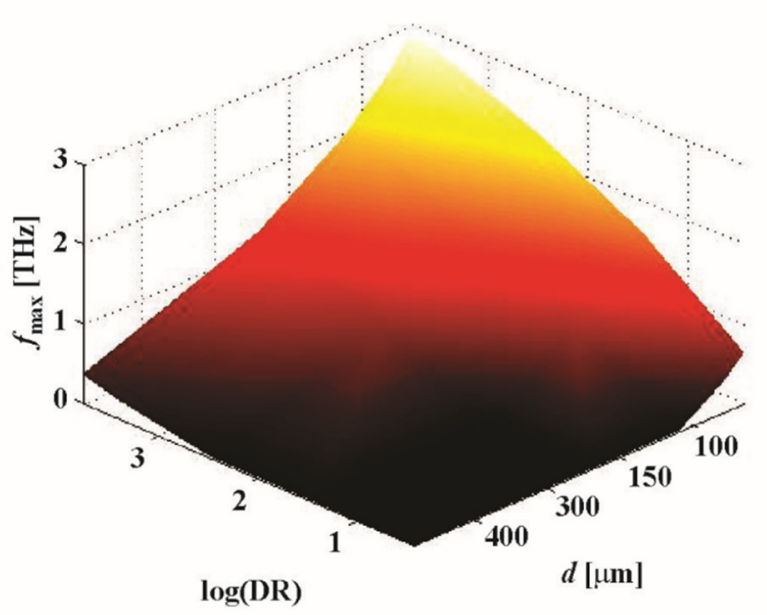

Figure 4. (a) The absorption coefficient versus frequency is shown for a representative case of $120 \mu \mathrm{m}$ microfluidic platform thickness and 2.4E3 dynamic range. (b) The maximum measurable frequency is plotted against the $\log$ of the dynamic range and the microfluidic platform thickness. The maximum measurable frequency has a maximum value of approximately 3 $\mathrm{THz}$ and decreases with decreasing dynamic range and increasing microfluidic platform thickness.

can observe an increase of the maximum measurable frequency with decreased $d$ and increased DR. These results can be used to inform future designs.

\section{CONCLUSIONS}

In conclusion, for THz-TDS microfluidic systems, the microfluidic platform thickness and dynamic range of the THzTDS system play a critical rule in defining the maximum measurable frequency. It was found that the maximum measurable frequency decrease with decreasing the dynamic range and increasing sample thickness. These fundamental results can be used in the design of future microfluidic THz-TDS systems for biomedical applications.

\section{ACKNOWLEDGMENT}

The authors would like to acknowledge financial support through the Natural Science and Engineering Research Council of Canada.

\section{REFERENCES}

[1] H. Pahlevaninezhad, B. Heshmat, and T. E. Darcie, "Efficient terahertz slot-line waveguides," Opt. Express, vol. 19, no. 26, pp. B47-B55, 2011.

[2] X. Jin, C. M. Collier, J. J. A. Garbowski, B. Born, and J. F. Holzman, "Ultrafast transient responses of optical wireless communication detectors," Appl. Opt., vol. 52, no. 20, pp. 5042-5049, 2013.

[3] C. M. Collier, B. Born, X. Jin, and J. F. Holzman, "Ultrafast chargecarrier and phonon dynamics in GaP," Appl. Phys. Lett., vol. 103, no. 7, 072106(1-4), 2013

[4] S.-W. Huang et al., "Globally stable microresonator turing pattern formation for coherent high-power THz radiation on-chip," Phys. Rev. X, vol. 7, no. 4, 041002(1-10), 2017.

[5] C. M. Collier and J. F. Holzman, "Ultrafast photoconductivity of crystalline, polycrystalline and nanocomposite $\mathrm{ZnSe}$ material systems for terahertz applications," Appl. Phys. Lett., vol. 104, no. 4, 042101(15), 2014.

[6] M. Reid and R. Fedosejevs, "Terahertz birefringence and attenuation properties of wood and paper," Appl. Opt., vol. 45, no. 12, pp. 2766$2772,2006$.

[7] C. M. Collier, T. J. Stirling, I. R. Hristovski, J. D. A. Krupa, and J. F. Holzman, "Photoconductive terahertz generation from textured semiconductor materials," Sci. Rep., vol. 6, 23185(1-10), 2016

[8] J. F. Federici et al, "THz imaging and sensing for security applications-explosives, weapons and drugs," Semicon. Sci. Technol., vol.20, no.7, pp. S266-S280, June 2005.

[9] J. Federici, and L. Moeller, "Review of terahertz and subterahertz wireless communications," J. Appl. Phys., vol. 107, pp 111101-22, 2010 .

[10] B. B. Hu, and M. C. Nuss, "Imaging with terahertz waves," Opt. Lett., vol. 16, pp. 1716-1719, August 1995.

[11] A. Markelz, S. Whitmire, J. Hillebrecht, and R. Birge, "THz time domain spectroscopy of biomolecular conformational modes," Phys. Med. Biol., vol. 47, iss. 21, pp. 3797-3805, October 2002.

[12]C. M. Collier, M. H. Bergen, T. J. Stirling, M. A. DeWachter, and J. F. Holzman, "Optimization processes for pulsed terahertz systems," Appl. Opt., vol. 54, no. 3, pp. 535-545, 2015.

[13]A. N. Bogomazova et al., "No DNA damage response and negligible genome-wide transcriptional changes in human embryonic stem cells exposed to terahertz radiation," Sci. Rep., vol. 5, 7749, 2015.

[14]E. Pickwell et al., "In vivo study of human skin using pulsed terahertz radiation," Phys. Med. Biol., vol. 49, pp. 1595-1607, 2004.

[15] P. Y. Han, M. Usman, and S. Kono, "A direct comparison between terahertz time-domain spectroscopy and far-infrared Fourier transform spectroscopy," J. App. Phys., vol. 89, no. 4, pp. 2357-2363, February 2001.

[16] C.-C. Chen, D.-J. Lee, T. Pollock, and J. F. Whitaker, "Pulsedterahertz reflectometry for health monitoring of ceramic thermal barrier coatings," Opt. express, vol.18, iss.4, pp. 3477-3486, 2010.

[17] P. A. George et al, "Microfluidic devices for terahertz spectroscopy of biomolecules," Opt. Express, vol. 16, no. 3, pp. 1577-1582, January 2008 . 
[18] C. M. Collier, M. Wiltshire, J. Nichols, B. Born, E. L. Landry, and J. F. Holzman, "Nonlinear dual-phase multiplexing in digital microfluidic architectures," Micromachines, vol. 2, pp. 369-384, 2011.

[19]J. Nichols, C. M. Collier, E. L. Landry, M. Wiltshire, B. Born, and J. F. Holzman, "On-chip digital microfluidic architectures for enhanced actuation and sensing," J. Biomed. Opt., vol. 17, no. 6, 067005(1-7), 2012.

[20]J. Nichols, E. L. Landry, B. Born, M. Wiltshire, C. M. Collier, and J. F. Holzman, "Optical sensing for on-chip digital microfluidics," Proceedings of SPIE Photonics West, San Francisco, USA, January, 2012.

[21] M. Naftaly, and R. Dudley, "Methodologies for determining the dynamic ranges and signal-to-noise ratios of terahertz time-domain spectrometers," Opt. Lett., vol. 34, iss. 8, pp. 1213-1215, April 2009.

[22] G. Rodriguez and A. J. Taylor, "Screening of the bias field in terahertz generation from photoconductors," Opt. Lett., vol. 21, no. 14, pp. 1046-1048, July 1996.
[23] C. M. Collier, T. J. Stirling, S. Dekock-Kruger, and J. F. Holzman, "Spectral response tuning of photoconductive terahertz emitters with binary phase masks," IEEE J. Quantum Electron, vol. 23, iss. 4, JulyAugust 2017.

[24] P. Shumyatsky, and R. R. Alfano, "Terahertz sources," J. Biomed. Opt., vol. 16, iss. 3, 0330011, March 2011.

[25] P. U. Jepsen, and B. M. Fischer, "Dynamic range in terahertz timedomain transmission and reflection spectroscopy," Opt. Lett., vol. 30, iss. 1, pp. 29-31, January 2005. 